\title{
El género Rhytidhysteron (Dothideomycetes, Ascomycota) en México
}

\section{The genus Rhytidhysteron (Dothideomycetes, Ascomycota) in Mexico} Acta Botanica Mexicana

\author{
Aurora Cobos-Villagrán' (1D , César Hernández-Rodríguez² (D) , Ricardo Valenzuela ${ }^{3}$ (D) , Lourdes Villa-Tanaca² (D) , Rosa \\ Paulina Calvillo-Medina ${ }^{4}$ (1) , Luz Elena Mateo-Cid ${ }^{5}$ (1) , Michelle Martínez-Pineda ${ }^{3}$ (1) , Tania Raymundo 3,6 (1)
}

\section{Resumen:}

Antecedentes y Objetivos: Actualmente, se reconocen 21 especies adscritas al género Rhytidhysteron. En México sólo se ha reportado Rhytidhysteron rufulum. El género se caracteriza por sus histerotecios naviculares a apoteciales, el borde es estriado o liso; los colores del epitecio varían de anaranjado-rojizo, amarillo, verde a negros y las ascosporas presentan tres septos longitudinales. El objetivo del presente estudio es realizar una revisión taxonómica del género Rhytidhysteron en México, basada en caracteres macro y micromorfológicos.

Métodos: Se realizaron diez exploraciones entre 2017 y 2019 en diversas regiones de México. Además, se revisaron los especímenes depositados en la colección de hongos del herbario ENCB. Los ejemplares fueron estudiados, descritos y determinados de acuerdo con las técnicas tradicionales en micología.

Resultados clave: Se revisaron 381 especímenes pertenecientes al género Rhytidhysteron, de los cuales se determinaron cuatro especies: Rhytidhysteron neohysterinum, una nueva especie para la ciencia, $R$. neorufulum y $R$. thailandicum que son nuevos registros para el país, y $R$. rufulum previamente citado.

Conclusiones: El género Rhytidhysteron tiene una alta diversidad de especies en México. La mayoría de los especímenes se han encontrado sobre ramas en descomposición, principalmente en géneros de la familia Fabaceae en bosque tropical caducifolio, lo que podría sugerir que este grupo de plantas representa el microhábitat al que las especies de Rhytidhysteron se han adaptado.

Palabras clave: distribución, histerotecio, Hysteriales, nueva especie, taxonomía.

\section{Abstract:}

Background and Aims: Currently, 21 species associated with the genus Rhytidhysteron are recognized. In Mexico, only Rhytidhysteron rufulum has been reported. The genus is characterized by its navicular to apotecial histerothecia, the margin is striate or smooth; the colours of the epithecium vary from orange-reddish, yellow, green to black and its ascospores present three longitudinal septa. The objective of this study is to carry out a taxonomic review of the genus Rhytidhysteron in Mexico, based on macro- and micromorphological characters.

Methods: Ten exploration trips were realized between 2017 to 2019 in several regions of Mexico. In addition, the specimens deposited in the fungus collection of the ENCB herbarium were reviewed. The specimens were studied, described and determined according to traditional mycological techniques.

Key results: 381 specimens belonging to the genus Rhytidhysteron were reviewed, of which four species were determined: $R$. neohysterinum, a new species for science, $R$. neorufulum and $R$. thailandicum, new records for the country, and the previously cited $R$. rufulum.

Conclusions: The genus Rhytidhysteron has a high diversity of species in Mexico. Most specimens have been found on decomposing branches, mainly of genera of the Fabaceae family in tropical dry forest, which suggest that this group of plants represent the microhabitat to which Rhytidhysteron species have best adapted.

Key words: distribution, Hysteriales, hysterothecium, new species, taxonomy.

'Maestría en Biociencias, Posgrado en Biociencias, Escuela Nacional de Ciencias Biologicas, Instituto Politécnico Nacional, $11340 \mathrm{Cd}$. Mx., México.

${ }^{2}$ Instituto Politécnico Nacional, Escuela Nacional de Ciencias Biológicas, Departamento de Microbiología, Laboratorio de Biología Molecular de Bacterias y Levaduras, $11340 \mathrm{Cd}$. Mx., México.

${ }^{3}$ Instituto Politécnico Nacional, Escuela Nacional de Ciencias Biológicas, Departamento de Botánica, Labo ratorio de Micología, 11340 Cd. Mx., México.

${ }^{4}$ Universidad Autónoma de Querétaro, Facultad de Química, Laboratorio de Microbiología Molecular 76010 Qro., México.

Instituto Politécnico Nacional, Escuela Nacional de Ciencias Biológicas, Departamento de Botánica, Laboratorio de Ficología, 11340 Cd. Mx., México.

${ }^{6}$ Autor para la correspondencia: traymundoo@ipn.mx
Recibido: 11 de febrero de 2020

Revisado: 25 de marzo de 2020.

Aceptado por Marie-Stéphanie Samain: 4 de mayo de 2020

Publicado Primero en línea: 15 de mayo de 2020.

Publicado: Acta Botanica Mexicana 127(2020).
Citar como: Cobos-Villagrán, A., C. H. Hernández-Rodríguez, R. Valenzuela, L. Villa-Tanaca, R. P. CalvilloMedina, L. E. Mateo-Cid, M. Martínez-Pineda y T. Raymundo. 2020. El género Rhytidhysteron (Dothideomycetes, Ascomycota) en México. Acta Botanica Mexicana 127: e1675. DOI: 10.21829/abm127.2020.1675 


\section{Introducción}

Rhytidhysteron fue descrito por Spegazzini (1881); es un género que pertenece a la familia Hysteriaceae, orden Hysteriales (Boehm et al., 2009a, 2009b), clase Dothideomycetes, phylum Ascomycota. Las características diagnósticas son: histerotecio elíptico o irregular, con apertura lenticular o irregular cuando húmedo, perpendicularmente estriado o no a lo largo del eje largo, negro, rojo o amarillo en el epitecio, cuando está seco el margen se dobla, formando una hendidura alargada; peridio compuesto de 1-2 capas, capa externa de marrón oscuro a negro, células de textura globulosa-angular, radial, capa interna de células hialinas a ligeramente pigmentadas de textura globulosa-angular a prismática; hamatecio denso, septado, pseudoparáfisis, ramificado y formando un epitecio oscuro sobre las ascas, fusionado y ligeramente ensanchado en el ápice, encerrado en una matriz gelatinosa; ascas con 6-8 esporas bitunicadas, cilíndricas, redondeadas en el ápice, con una cámara ocular distinta; ascosporas uniseriadas, se superponen ligeramente, de forma elipsoidal a fusiforme, ligeramente redondeadas o puntiagudas en ambos extremos, 1-3-septos, constreñidos en el tabique central, de color marrón rojizo a marrón, sin una vaina mucilaginosa. Hasta ahora se han descrito 21 especies (Méndez-Mayboca et al., 2010; Almeida et al., 2014; Álvarez et al., 2016; Thambugala et al., 2016; Soto-Medina y Lücking, 2017; Kumar et al., 2019).

El género Rhytidhysteron es de amplia distribución en zonas tropicales y subtropicales del planeta (Samuels y Müller, 1979). Rhytidhysteron rufulum (Spreng.) Speg. es la especie mayormente citada en diversas regiones del mundo: Argentina, Brasil, China, Costa Rica, Cuba, Dominica, Estados Unidos de América, Filipinas, Francia, Ghana, India, Islas Cook, Jamaica, Japón, Kenia, Malasia, Micronesia, Nueva Guinea, Nueva Zelanda, Puerto Rico, Sierra Leona, Tanzania, Taiwán y Tonga (Almeida et al., 2014; Chen y Hsieh, 1996; Murillo et al., 2009). Recientemente, se ha citado de las Islas Andamán, India (Niranjan, et al., 2018). En México, sólo se ha citado a $R$. rufulum de los estados de Sonora (Méndez-Mayboca et al., 2008, 2010), Oaxaca (Raymundo et al., 2014; Álvarez et al., 2016), Chiapas, Guerrero, Nuevo León, Puebla, Querétaro, Quintana Roo,
Sonora, Tabasco, Tamaulipas, Veracruz y Yucatán (Chacón et al., 2014). El presente estudio tiene como objetivo describir las especies de Rhytidhysteron que se distribuyen en México.

\section{Materiales y Métodos}

Para el presente trabajo se revisó el herbario ENCB de la Escuela Nacional de Ciencias Biológicas del Instituto Politécnico Nacional (IPN). Además, se realizaron exploraciones de 2017 a 2019 en diversas regiones del país. Todo el material se examinó siguiendo las técnicas tradicionales en micología. Se tomaron fotografías empleando una cámara digital (Nikon, D7000, Tokio, Japón) con lente macro de $85 \mathrm{~mm}$ (Nikon, Tokio, Japón). Los ejemplares recolectados se caracterizaron en fresco tomando los datos morfológicos de los histerotecios como tamaño, forma, color, características del epitecio, hábito de crecimiento y hábitat. Los histerotecios se midieron en un microscopio estereoscópico (Zeiss 475002, Jena, Alemania). Se realizaron cortes transversales en la parte media del histerotecio y se montaron en preparaciones temporales en alcohol al $70 \%$ y con $\mathrm{KOH}$ al $10 \%$; se observaron al microscopio óptico (Zeiss K-7, Jena, Alemania) para describir las características de la textura y grosor del peridio; tamaño, forma y diámetro de las paráfisis; tamaño, forma, número y disposición de las ascosporas en el asca; tamaño, forma, color y número de septos de ascosporas; en estas últimas se midieron 30 para obtener la media. Las especies se determinaron con las claves especializadas de Thambugala et al. (2016) y Soto-Medina y Lücking (2017), principalmente.

\section{Resultados}

Se determinaron cuatro especies delgénero Rhytidhysteron: $R$. rufulum reportado previamente, $R$. neorufulum Thambug. \& K.D. Hyde y R. thailandicum Thambug. \& K.D. Hyde se citan por primera vez para México; $R$. neohysterinum sp. nov. se describe como especie nueva para la ciencia. Rhytidhysteron rufulum es la especie mejor distribuida en bosque tropical caducifolio, mientras $R$. thailandicum está mejor representada en matorral xerófilo. La clasificación seguida en este trabajo es de acuerdo con Kirk et al. (2008). 


\section{Taxonomía}

Rhytidhysteron neohysterinum Cobos-Villagrán, Hdz.-Rdz.,

R. Valenz. \& Raymundo sp. nov. Fig. 1.

TIPO: MÉXICO. San Luis Potosí, municipio Guadalcázar, Carretera 75-D, San Luis - Cd. Valles km 98, 02.XI.2017, A. Cobos-Villagrán 775 (holotipo: ENCB). Mycobank: MB 835584.

Differunt per Rhytidhysteron hysterinum (Dufour) Samuels \& E. Müll. Hysterothecia (1.5-)1.9-2.3(-2.5) mm longa, (0.7-)1.0-1.6(-2.2) mm latum, (0.7-)0.9-1.1 mm altum ( $\bar{x}=1.97 \times 1.32 \times 0.9 \mathrm{~mm}, n=10)$; epithecium luteum, ad mutationes in purpura KOHe 10\%; bitunicati ascis 160 185 нm; ascosporae (23-) 24.8-29(-31) × (8.5-)8.8-10(-11.2) $\mu m(\bar{x}=26.6 \times 9.4 \mu m, n=30)$. Ad lignis in Fabaceae in sylvis tropical caducifoliae.

Histerotecios (1.5-)1.9-2.3(-2.5) mm de largo, (0.7)1.0-1.6(-2.2) mm de ancho, (0.7-) $0.9-1.1 \mathrm{~mm}$ de alto $(\bar{x}=1.97 \times 1.32 \times 0.9 \mathrm{~mm}, \mathrm{n}=10)$, apoteciales, rectos o flexuosos cuando jóvenes, con los extremos agudos, negros, carbonosos, superficiales, solitarios, raramente gregarios, borde estriado con estrías muy separadas entre sí (anchas), con surco o hendidura longitudinal y se vuelve lenticular (cuando maduros o con la humedad), abriendo de forma irregular, raramente abre de forma apotecial, exponiendo el himenio; peridio de un grosor regular de 52-68 $\mu \mathrm{m}$, ensanchando ligeramente hacia la parte basal hasta 77 $\mu \mathrm{m}$, compuesto por capas de células pseudoparenquimáticas, color marrón a negro, de textura isoradiada o prismáticas, con células de 9.2-11.8 × 7-9 $\mu \mathrm{m}$, paredes delgadas, $1 \mu \mathrm{m}$ de grosor; epitecio anaranjado cambia a morado en $\mathrm{KOH}$ al $10 \%$; pseudoparáfisis 2-3 $\mu \mathrm{m}$ de ancho, capitadas, filamentosas, densas, hialinas, septadas, sin ramificar, fuertemente anastomosadas por encima de las ascas formando el epitecio; ascas bitunicadas, 160-185 × 12-13 $\mu \mathrm{m}$, clavadas a cilíndricas, hialinas, uniseriadas, octospóricas, con la base corta y ligeramente sinuosa; ascosporas (23-)24.8$29(-31) \times(8.5-) 8.8-10(-11.2) \mu \mathrm{m}(\overline{\mathrm{x}}=26.6 \times 9.4 \mu \mathrm{m}, \mathrm{n}=30)$, elipsoides, redondeados en ambos extremos, color pardo a marrón claro, con un septo transversal central, cinguladas, pared gruesa y lisa.

Material estudiado: MÉXICO. San Luis Potosí, municipio Guadalcázar, carretera 75-D, San Luis - Cd. Valles km 98, 02.XI.2017, A. Cobos-Villagrán 773 (ENCB), 776 (ENCB), 777 (ENCB). Municipio Tamasopo, Potrero del Hotel Real Kimera, ejido Los Cafetales, 03.XI.2017, A. Cobos-Villagrán 786 (ENCB).

Hábitat: dispersos, sobre madera en descomposición de Acacia Mill. y otras leguminosas sin identificar en el matorral xerófilo y bosque tropical caducifolio.

Distribución: Esta especie solo se conoce de la localidad tipo (Altiplanicie) y Tamasopo, San Luis Potosí (Sierra Madre Oriental).

Etimología: hace referencia a Rhytidhysteron hysterinum por las características similares.

Notas taxonómicas: Rhytidhysteron neohysterinum se caracteriza por presentar en las ascosporas un solo septo transversal. Se diferencia de $R$. hysterinum porque las ascosporas son ligeramente más pequeñas. En $R$. neohysterinum son de (23-)24.8-29 × (8.5-)8.8-10(-11.2) $\mu \mathrm{m}$ en comparación con $R$. hysterinum 22-32 × 10-16 $\mu \mathrm{m}$ (Sierra-López, 2006) y 21.58-28.35(-36.39) × (9.97-)10.98-11.95(-14.17) $\mu \mathrm{m}$ (como Eutryblidiella hysterina (Dufour) Petr. (Merino y Tello-Mora, 2013) que son más anchas. Otro aspecto es que $R$. hysterinum solo se distribuye en España (Sierra-López, 2006; Merino y Tello-Mora, 2013) y Francia (Samuels y MüIler, 1979; Boehm et al., 2009b), y únicamente se ha reportado sobre madera de Buxus spp.

Rhytidhysteron neorufulum Thambug. \& K.D. Hyde, Cryptogamie, Mycologie 37(1): 110. 2016. TIPO: TAILANDIA. Provincia de Chiang Rai, Centro de Investigación Hortícola Chiangrai, 2012, K. M. Thambugala 3 (holotipo: MFLU, isotipo: PDD). Fig. 2. 


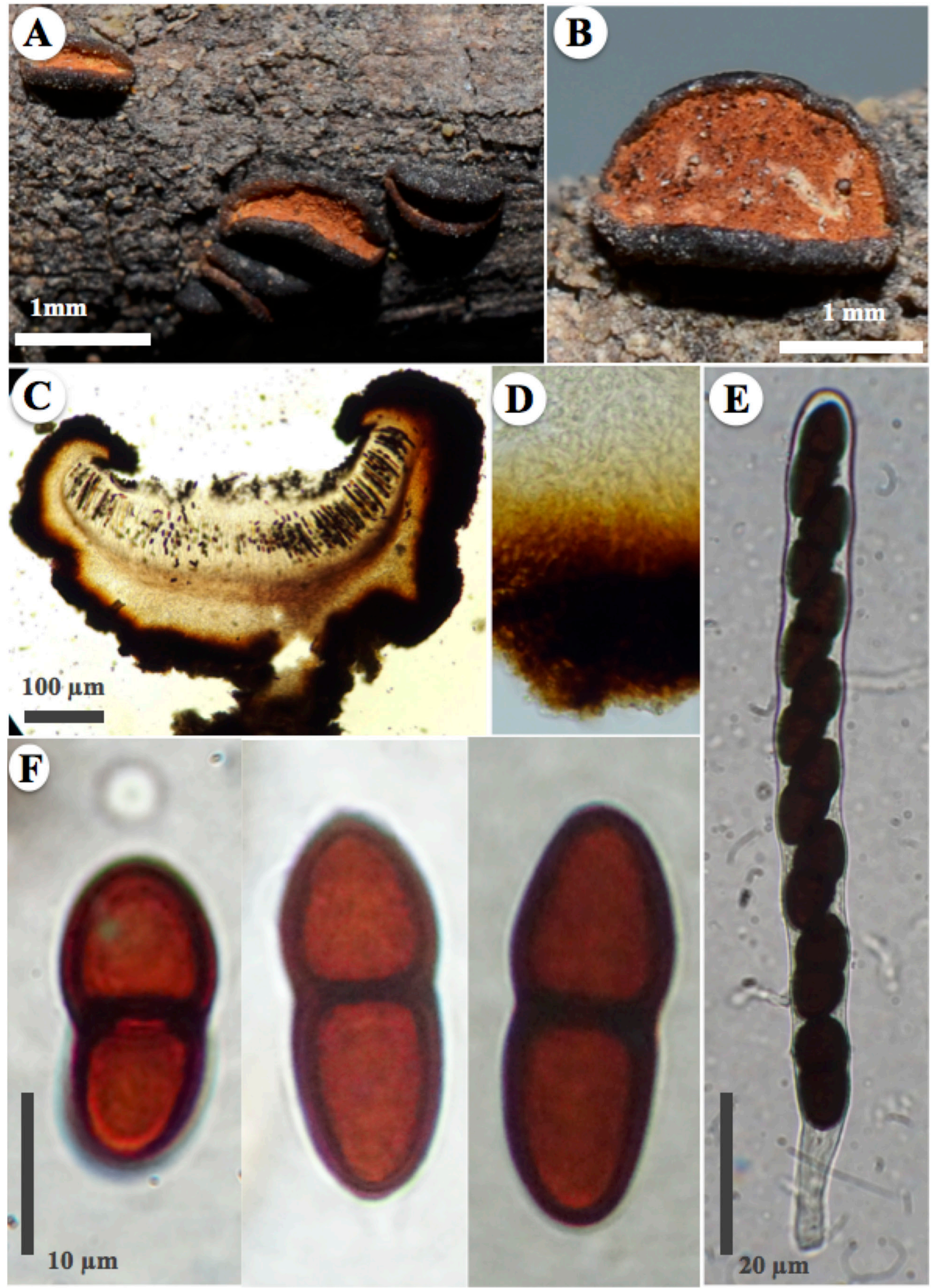

Figura 1: Rhytidhysteron neohysterinum Cobos-Villagrán, Hdz.-Rdz., R. Valenz. \& Raymundo. A. histerotecios; B. epitecio naranja y margen estriado; C. corte transversal del histerotecio; D. células del peridio; E. asca; F. ascosporas. 


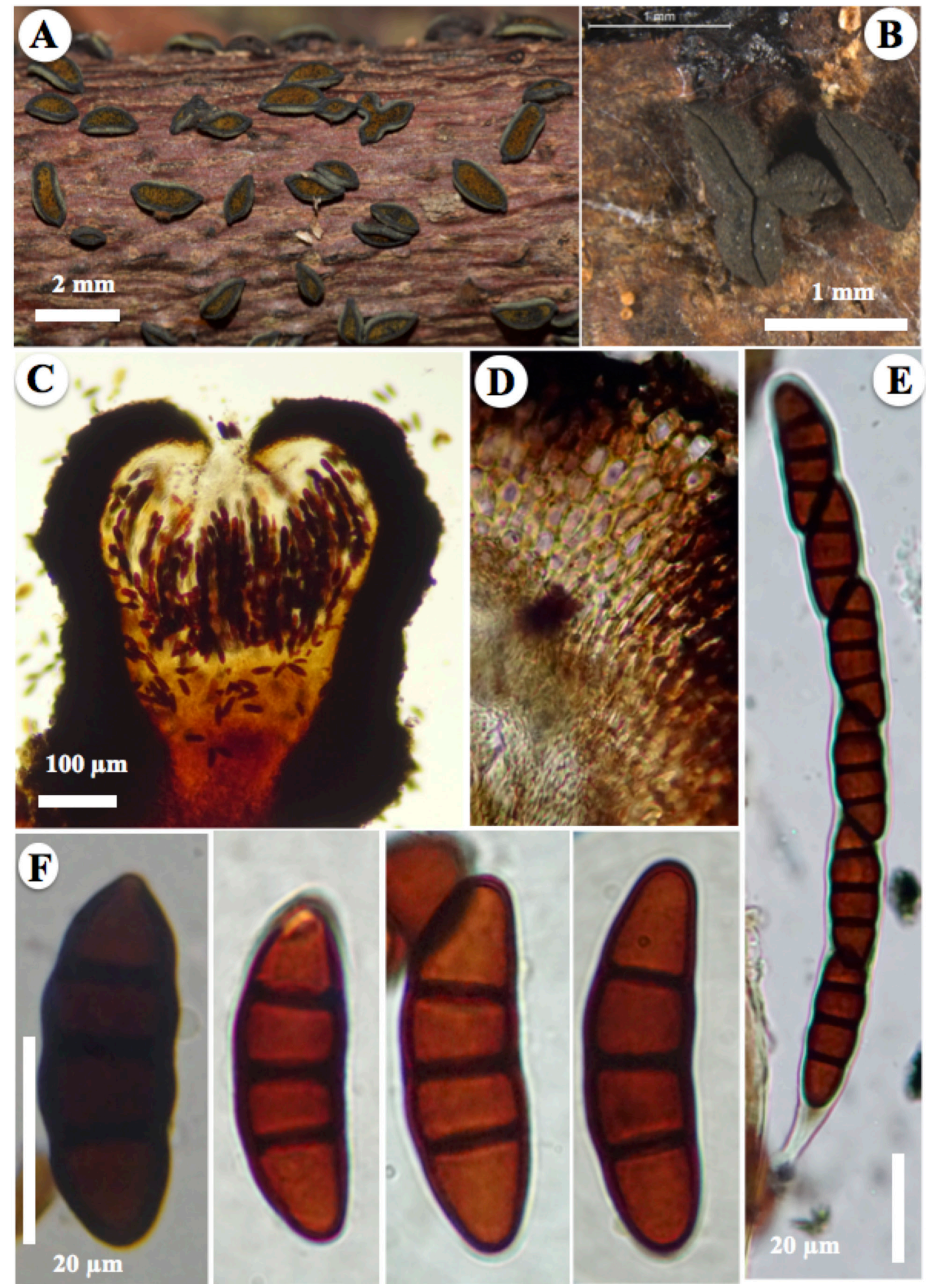

Figura 2: Rhytidhysteron neorufulum Thambug. \& K.D. Hyde. A. histerotecios; B. margen liso; C. corte transversal del histerotecio; D. células del peridio; E. asca; F. ascosporas. 
Histerotecios (1.9-)2.3-2.8(-3) mm de largo, 0.7-1 $\mathrm{mm}$ de ancho, (0.5-)1.1-1.3 mm de alto $(\bar{x}=2.48 \times 0.87 \times$ $1.01 \mathrm{~mm}, \mathrm{n}=10$ ), naviculares alargados a fusiformes, en ocasiones triradiados, negros a verde opaco, carbonosos a coriáceos, superficiales, solitarios a gregarios, margen liso, sin embargo presenta unas líneas a lo largo del ascoma de forma paralela al borde del histerotecio, con surco o hendidura longitudinal y se vuelve lenticular (cuando maduros o con la humedad), abriendo de forma irregular a discoidal, tomando la forma apotecial, exponiendo el himenio; peridio de un grosor entre 50-60 $\mu \mathrm{m}$, adelgazándose ligeramente hacia la parte apical y ensanchándose hacia la base hasta $250 \mu \mathrm{m}$, compuesto por capas de células pseudoparenquimáticas, color marrón oscuro a negro en la parte superior, de textura isoradiada o prismáticas con células de (7-)10-13 $(-15) \times 6-7(-9) \mu \mathrm{m}$, paredes gruesas, $1 \mu \mathrm{m}$ de grosor; epitecio anaranjado a anaranjado rojizo, cambia a violeta en $\mathrm{KOH}$ al 10\%; pseudoparáfisis 1-2 $\mu \mathrm{m}$ de ancho, ensanchándose hacia la parte apical hasta $4 \mu \mathrm{m}$, capitadas, filamentosas, densas, hialinas, sin septos, sin ramificar, fuertemente anastomosadas por encima de las ascas formando el epitecio; ascas bitunicadas, $238 \times 15 \mu \mathrm{m}$, claviformes a cilíndricas, hialinas, uniseriadas, octospóricas, con la base corta y recta; ascosporas (28.8-)31.2-33.6(-36.8) × (10.4-)11.2-12 $\mu \mathrm{m}(\overline{\mathrm{x}}=32 \times 11 \mu \mathrm{m}, \mathrm{n}=30)$, elipsoidales a fusiformes, ligeramente redondeadas o puntiagudas en ambos extremos, amarillentas cuando están inmaduras, color marrón rojizo a marrón cuando maduran, con tres septos longitudinales, cinguladas, pared gruesa y lisa.

Material estudiado: MÉXICO. Campeche, municipio Calakmul, Reserva de la Biósfera Calakmul, 4.XII.2010, T. Raymundo 3622 (ENCB), s.n. (ENCB); loc. cit., R. Valenzuela 14153 (ENCB), 14153a (ENCB). Municipio Champotón, autopista Champotón - Campeche km 180, 19.I.2018, T. Raymundo 7234 (ENCB). Colima, municipio Manzanillo, Oeste de la Bahía de Santiago, carretera a Barra de Navidad, 26.X.1967, G. Guzmán 6232 (ENCB); humedales, a 4 km al N de Manzanillo, 12.VII.2016, A. Camargo s.n. (ENCB); loc. cit., R. Valenzuela 16708 (ENCB), 16717 (ENCB), 16718 (ENCB), 16719 (ENCB), 16729 (ENCB), 16732 (ENCB), 16747 (ENCB). Jalisco, municipio La Huerta, Reserva de la Biósfera Chamela Cuixmala, Estación Biológica Chamela
IBUNAM, Camino Búho, 23.IX.2012, T. Raymundo 4449 (ENCB); Camino Bosque de la Enseñanza, 27.IX.2013, I. Álvarez 519 (ENCB), 522 (ENCB); loc. cit., 15.IX.2018, A. Cobos-Villagrán 2033 (ENCB), 2034 (ENCB), 2035 (ENCB), $2035 a$ (ENCB), 2038 (ENCB), 2039 (ENCB), 2040 (ENCB), 2041 (ENCB), 2050 (ENCB), 2056 (ENCB), 2059 (ENCB); loc. cit., A. Gay AG15091813 (ENCB); loc. cit., S. Bautista-Hernández 921 (ENCB), 925 (ENCB); loc. cit., A. HernándezZamora 319 (ENCB); loc. cit., T. Raymundo 7837 (ENCB), 7844 (ENCB); loc. cit., R. Valenzuela 18533 (ENCB), 18538 (ENCB); Camino Chachalacas 16.IX.2018, A. Cobos-Villagrán 2066 (ENCB); Camino Eje Central, 22.IX.2012, T. Raymundo 4431 (ENCB), 4434 (ENCB), 4442 (ENCB); loc. cit., 28.IX.2013, I. Álvarez 535 (ENCB), 536 (ENCB), 537 (ENCB); loc. cit., 16.IX.2018, A. Cobos-Villagrán 2057 (ENCB), 2058 (ENCB); loc. cit., A. Hernández-Zamora 326 (ENCB), 341 (ENCB); Camino Tejón, 16.IX.2018, A. Cobos-Villagrán 2062 (ENCB), 2063 (ENCB). Nayarit, municipio San Blas, Carretera Tepic - San Blas km 27, 29.IX.2018, M. Sánchez 1384 (ENCB); loc. cit., T. Raymundo 7963 (ENCB), 7977 (ENCB); loc. cit., R. Valenzuela 18667 (ENCB). Municipio Xalisco, Cerro San Juan, Reserva de la biósfera estatal San Juan Xalisco, 28.IX.2018, M. Sánchez 1305 (ENCB); loc. cit., T. Raymundo 7908 (ENCB), 7909 (ENCB). Oaxaca, municipio San Pedro Tututepec, Juquila, km 7.2 del camino Los Azufres - La Grúa, Parque Nacional Lagunas de Chacahua, 12.XI.2011, T. Raymundo 4119 (ENCB); km 5 del camino Los Azufres - La Grúa, 12.XI.2013, D. Castro-Bustos 249 (ENCB). Quintana Roo, municipio Cozumel, Centro de conservación y educación ambiental, Parque Museo de Cozumel, 19.I.2018, A. Cobos-Villagrán 915 (ENCB); alrededores del Transcaribe API, 21.I.2018, A. Cobos-Villagrán 960 (ENCB), 961 (ENCB), 963 (ENCB); loc. cit., T. Raymundo 7346 (ENCB); loc. cit., R. Valenzuela 18019 (ENCB), 18022 (ENCB), 18025 (ENCB), $18025 a$ (ENCB); zona arqueológica San Gervasio, Chen-tuk, 21.I.2018, T. Raymundo 7337 (ENCB); loc. cit., 17.VI.2018, A. Bernal 132 (ENCB); loc. cit., A. Cobos-Villagrán 1840 (ENCB), 1841 (ENCB), 1843 (ENCB); Parque Ecológico Punta Sur, 16.VI.2018, A. CobosVillagrán 1816 (ENCB). San Luis Potosí, municipio Tamasopo, carretera Río Verde - Cd. Valles km 6414, VI.2009, E. Nolasco y F. González 8 (ENCB); Potrero del Hotel Real Kimera, ejido Los Cafetales, 03.XI.2017, A. Cobos-Villa- 
grán 787 (ENCB), 790 (ENCB), 791 (ENCB). Tabasco, municipio Emiliano Zapata, UMA Los Manatíes, 19.VI.2015, E. Escudero-Leyva 484 (ENCB); loc. cit., R. Valenzuela 15609 (ENCB), s.n. (ENCB); loc. cit., 20.VI.2015, E. EscuderoLeyva s.n. (ENCB). Tamaulipas, municipio Gómez Farías, La Florida, Reserva de la Biósfera El Cielo, 05.X.2017, A. Cobos-Villagrán 534 (ENCB); loc. cit., T. Raymundo 6803 (ENCB), 6804 (ENCB), 6813 (ENCB); loc. cit., R. Valenzuela 17412 (ENCB), 17413 (ENCB), 17423 (ENCB); loc. cit., 24.VI.2018, A. Cobos-Villagrán 1861 (ENCB); loc. cit., 25.VI.2018, A. Cobos-Villagrán 1904 (ENCB), 1917 (ENCB); loc. cit., 26.VI.2018, A. Cobos-Villagrán 1932 (ENCB), 1949 (ENCB), 1950 (ENCB), 1951 (ENCB).

Hábitat: solitario a gregario, sobre madera de Bursera Jacq. ex L. en descomposición, en bosque tropical caducifolio en un gradiente altitudinal de 0 a $190 \mathrm{~m}$ y ocasionalmente hasta 1090 m s.n.m., causando una pudrición blanda.

Distribución: esta especie ha sido descrita recientemente de Chiang Rai Province, Tailandia (Thambugala et al., 2016) e Islas Andamán, India (Niranjan et al., 2018). En el presente estudio se registra por primera vez para México en las regiones fisiográficas Costa del Golfo de México, Costa Pacífica, Península de Yucatán y Sierra Madre Oriental.

Notas taxonómicas: Rhytidhysteron neorufulum se distingue fácilmente del resto de las especies porque el borde del margen de los histerotecios es completamente liso con células que presentan textura isoradiada. Su epitecio de color anaranjado cambia violeta en $\mathrm{KOH}$ al $10 \%$. Los especímenes mexicanos presentan las mismas características morfológicas que los descritos de Tailandia (Thambugala et al., 2016).

Rhytidhysteron rufulum (Spreng.) Speg., Anales Sociedad Científica Argentina 90(1-6): 177. 1921. TIPO: PUERTO RICO. C. Sprengel s.n. (Espécimen tipo no designado ni se menciona en que herbario se depositó). Fig. 3.

= Hysterium rufulum Spreng., Kongl. Svenska Vetenskaps Academiens Handlingar 41: 50. 1820.
$=$ Tryblidiella rufula (Spreng.) Sacc. Sacc., Syll. Fung. (Abellini) 2: 757. 1883.

= Triblidium rufulum (Spreng.) Ellis \& Everh., N. Amer. Pyren. (Newfield): 690. 1892.

= Brunaudia rufula (Spreng.) Kuntze (as 'Bruneaudia'), Revis. Gen. PI. (Leipzig) 3(3): 447. 1898.

Histerotecios 1-3 mm de largo, 0.5-1.5 mm de ancho, 0.5-1 mm de alto ( $\bar{x}=2 \times 1.12 \times 0.94 \mathrm{~mm}, \mathrm{n}=10)$, naviculares, alargados, rectos o flexuosos cuando jóvenes, con los extremos agudos, algunos son triradiados, negros, carbonosos, errumpentes o superficiales, solitarios o gregarios, borde estriado, con surco o hendidura longitudinal y se vuelve lenticular (cuando maduros o con la humedad), abriendo de forma irregular a discoidal, tomando la forma apotecial de 2-4 mm de diámetro, exponiendo el himenio; peridio 40$72 \mu \mathrm{m}$, adelgazándose hacia la parte media y basal, ensanchándose hacia la parte apical hasta $100 \mu \mathrm{m}$, compuesto por capas de células pseudoparenquimáticas, color marrón a marrón oscuro, de textura isodiamétrica o globulosa-angular, con células de 9.6-12 × 8-8.8 $\mu \mathrm{m}$, paredes gruesas, 1.6-2.4 $\mu \mathrm{m}$ de grosor; epitecio anaranjado-rojizo, amarillo o negro cambia a magenta con $\mathrm{KOH}$ al $10 \%$; pseudoparáfisis 1.6-2.4 $\mu \mathrm{m}$ de ancho, ensanchándose hacia la parte apical hasta 3.2-4 $\mu \mathrm{m}$, capitadas, filamentosas, densas, hialinas, septadas, ramificadas hacia el ápice, fuertemente anastomosadas por encima de las ascas formando el epitecio; ascas bitunicadas, 142.5-169.7 × 12-14.4 $\mu \mathrm{m}$, cilíndricas, hialinas, uniseriadas, octospóricas, con la base corta y sinuosa; ascosporas $22.4-30.4 \times 8-9.6 \mu \mathrm{m},(\overline{\mathrm{x}}=26 \times 8.8 \mu \mathrm{m}, \mathrm{n}=30)$, elipsoides a oblongas, fusiformes, ligeramente curvadas, color marrón a marrón rojizo, con tres septos transversales, cinguladas, pared gruesa y lisa.

Material estudiado: MÉXICO. Campeche, municipio Calakmul, Reserva de la Biósfera Calakmul, 3.XII.2010, T. Raymundo 3573 (ENCB), 3594 (ENCB), 3643 (ENCB), loc. cit., R. Valenzuela 14156 (ENCB); loc. cit., 4.XII.2010, T. Raymundo 3617 (ENCB), 3646 (ENCB). Municipio Champotón, carretera Champotón - Cd. del Carmen km 70, 03.I.2018, T. Raymundo 7209 (ENCB), 7214 (ENCB), 7215 (ENCB), 7216 (ENCB), 7217 (ENCB), 7218 (ENCB), 7219 (ENCB); loc. cit., R. Valenzuela 17771 (ENCB), 17775 


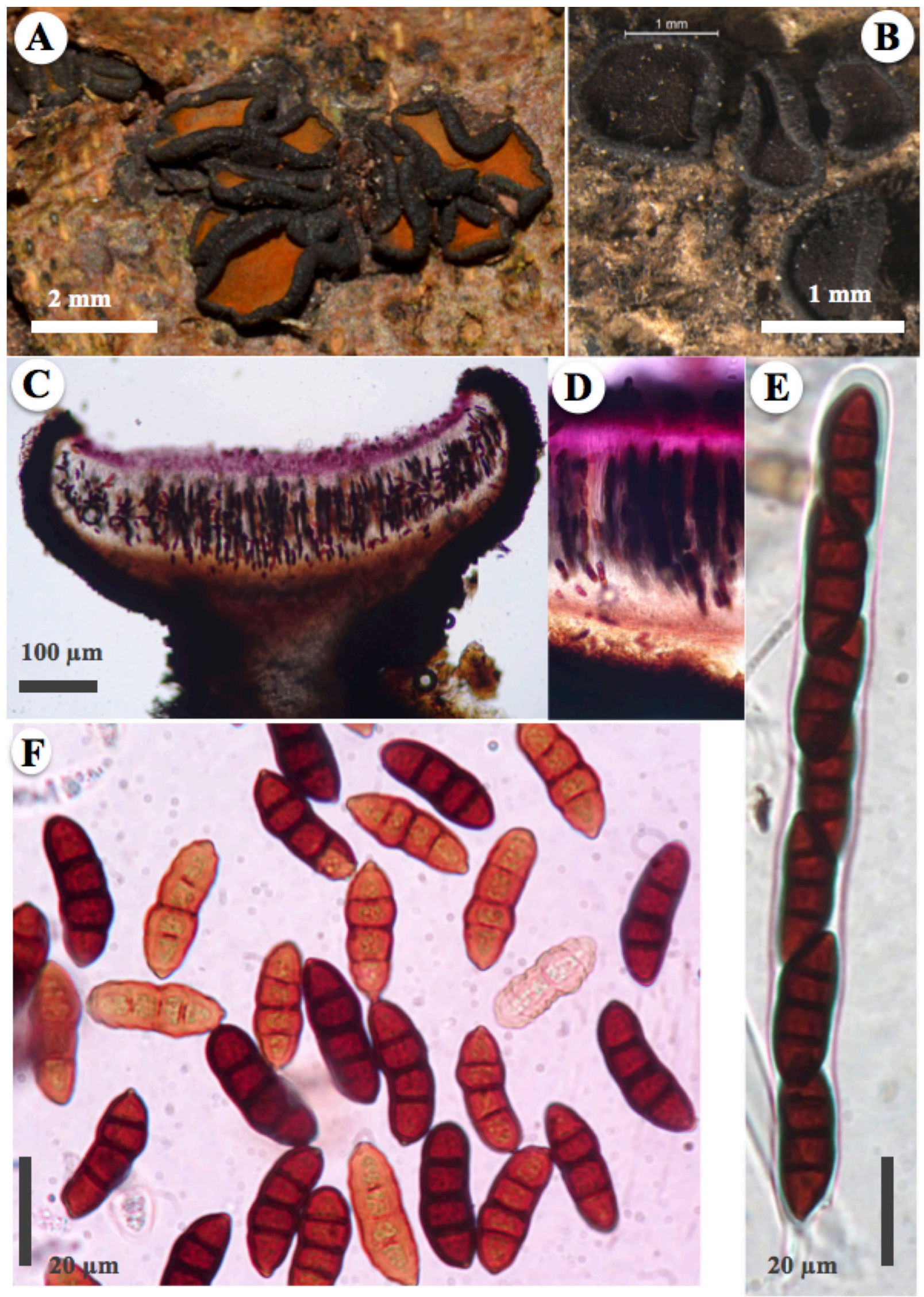

Figura 3: Rhytidhysteron rufulum (Spreng.) Speg. A. histerotecios; B. epitecio marrón vináceo y margen estriado; C. corte transversal del histerotecio; D. reacción magenta en $\mathrm{KOH}$ al 10\%; E. asca; F. ascosporas. 
(ENCB), 17777 (ENCB), 17779 (ENCB), 17781 (ENCB), 17783 (ENCB); autopista Champotón - Campeche km 180, 19.I.2018, A. Cobos-Villagrán 852 (ENCB), 853 (ENCB), 854 (ENCB), 855 (ENCB), 864 (ENCB); loc. cit., R. Valenzuela 17906 (ENCB). Estado de México, municipio Chiautla, Cerro Promesas, San Antonio Tepetitlán, 15.X.2017, M. Sánchez 1040 (ENCB), 1043 (ENCB), 1046 (ENCB), 1047 (ENCB). Municipio San Marcos Tecomaxusco, carretera Tecomaxusco, 03.X.2015, A. Barbosa 21 (ENCB). Hidalgo, municipio Huichapan, 28.V.2015, A. Cobos-Villagrán 29 (ENCB). Municipio Molango de Escamilla, Laguna Atezca, 01.VI.2018, T. Raymundo 4279 (ENCB), 4386 (ENCB), 7639 (ENCB); loc. cit., R. Valenzuela s.n. (ENCB). Guerrero, municipio Cocula, 27.VII.2011, R. Valenzuela 14355 (ENCB). Nayarit, municipio San Blas, Carretera Tepic - San Blas Km 31, 29.IX.2018, M. Sánchez 1328 (ENCB). Municipio Tepic, Camino a la Noria, Reserva de la biósfera estatal San Juan Xalisco, 28. IX.2018, M. Sánchez 1324 (ENCB). Municipio Xalisco, Cerro San Juan, Reserva de la Biósfera estatal San Juan Xalisco, 28.IX.2018, T. Raymundo 7910 (ENCB). Nuevo León, municipio Bustamante, Ojo de agua, 28.X.2017, A. Cobos-Villagrán 765 (ENCB), 766 (ENCB), 767 (ENCB), 768 (ENCB), 769 (ENCB). Municipio Sabinas Hidalgo, Sombreretillo, Sierra de Picachos, 27.X.2017, A. Cobos-Villagrán 682 (ENCB), 685 (ENCB), 686 (ENCB); camino de Sombreretillo a rancho La Sardina, Sierra de Picachos, 27.X.2017, A. Cobos-Villagrán 688 (ENCB), 689 (ENCB), 691 (ENCB), 692 (ENCB), 693 (ENCB), 695 (ENCB), 696 (ENCB), 697 (ENCB), 698 (ENCB), 703 (ENCB), 705 (ENCB), 706 (ENCB); faldas de la Sierra de Picachos, 27.X.2017, A. Cobos-Villagrán 715 (ENCB), 718 (ENCB), 719 (ENCB), 724 (ENCB), 726 (ENCB), 727 (ENCB), 729 (ENCB), 730 (ENCB), 732 (ENCB); loc. cit., T. Raymundo 7029 (ENCB); rancho La Sardina, Sierra de Picachos, 27.X.2017, A. Cobos-Villagrán 733 (ENCB); Parque La Turbina, La Cuchilla, 28.X.2017, A. Cobos-Villagrán 741 (ENCB), 744 (ENCB), 746 (ENCB), 747 (ENCB), 748 (ENCB), 749 (ENCB), 750 (ENCB), 751 (ENCB), 752 (ENCB), 756 (ENCB), 757 (ENCB), 762 (ENCB), 764 (ENCB); loc. cit., T. Raymundo 7049 (ENCB); loc. cit., R. Valenzuela 17569 (ENCB), 17588 (ENCB), 17589 (ENCB), 17603 (ENCB). Oaxaca, municipio Candelaria, Pochutla, Finca cafetalera "El Pacífico", 15. III.2014, D. Castro-Bustos 267 (ENCB). Municipio Ixtlán de Juárez, orilla del río Santa María, 02.III.2014, E. Escudero-
Leyva 374 (ENCB), 383 (ENCB), 385 (ENCB); Santa María Yahuiche, 02.III.2014, I. Ortega-López 226 (ENCB). Municipio San Miguel Santa Flor, Cuicatlán, Palomora, 07.VII.2014, D. Castro-Bustos 341 (ENCB); Llano mesa, 10.VIII.2015, A. Cobos-Villagrán 213 (ENCB). Municipio Santa María Chilchotla, Teotitlán, Cueva Blanca, San Miguel Nuevo, 09. VII.2014, T. Raymundo 5167 (ENCB). Municipio San Pedro Tututepec, Juquila, km 4 del camino Los Azufres - La Grúa, Parque Nacional Lagunas de Chacahua, 12.XI.2011, T. Raymundo 4095 (ENCB); loc. cit., R. Valenzuela 14595 (ENCB); km 5 del camino Los Azufres - La Grúa, Parque Nacional Lagunas de Chacahua, 12.XII.2011, I. Álvarez 336 (ENCB); loc. cit., T. Raymundo 4103 (ENCB), 4104 (ENCB); loc. cit., R. Valenzuela 14603 (ENCB), 14604 (ENCB); km 7.2 del camino Los Azufres - La Grúa, Parque Nacional Lagunas de Chacahua, 12.XI.2011, T. Raymundo 4115 (ENCB), 4118 (ENCB), 4140 (ENCB); loc. cit., R. Valenzuela 14615 (ENCB), 14618 (ENCB), 14619 (ENCB); loc. cit., C. Salinas 34 (ENCB); loc. cit., K. Victoria 24 (ENCB); loc. cit., 02.XI.2013, R. Valenzuela 15339 (ENCB). Municipio Santiago Laollaga, Istmo de Tehuantepec, Cerro Negro, 25.X.2014, E. Escudero-Leyva 38 (ENCB); loc. cit., M. Ramírez-Aguirre 12 (ENCB); loc. cit., R. Valenzuela 15991 (ENCB), 15992 (ENCB), 15993 (ENCB), 15994 (ENCB), 16063 (ENCB). Municipio Vega de Sol, rancho Gavilán, Tuxtepec, 01.II.2014, I. Ortega-López 220 (ENCB); El Zuzul, Tuxtepec, E. Escudero-Leyva s.n. (ENCB). Querétaro, municipio Arroyo seco, Puente de Ayutla, 16.X.2016, M. Contreras 1005 (ENCB). Quintana Roo, municipio Cozumel, Centro de conservación y educación ambiental, Parque Museo de Cozumel, 19.I.2018, A. Cobos-Villagrán 915 (ENCB); alrededores del Transacribe API, 21.I.2018, T. Raymundo 7346 (ENCB), 7348 (ENCB); loc. cit., R. Valenzuela 18024 (ENCB); Parque Ecológico Punta Sur, 16.VI.2018, A. Cobos-Villagrán 1811 (ENCB), 1817 (ENCB), 1820 (ENCB), 1821 (ENCB), 1823 (ENCB), 1824 (ENCB), 1825 (ENCB); loc. cit., T. Raymundo 7663 (ENCB); zona arqueológica San Gervasio, Chen-tuk, 17. VI.2018, A. Cobos-Villagrán 1844 (ENCB), 1845 (ENCB), 1861 (ENCB). San Luis Potosí, municipio Aquismón, Río Tampaón, alrededores de la Cascada Tamul, 03.XI.2018, T. Raymundo 8149 (ENCB); loc. cit., S. S. Salazar 39 (ENCB); loc. cit., $R$. Valenzuela 18849 (ENCB). Municipio El Naranjo, Cerro al N de Micos, Ciudad Valles, 29.XI.2012, I. Álvarez 
328 (ENCB), 329 (ENCB), 330 (ENCB). Municipio Guadalcázar, carretera Cerritos - entronque a Tula km 26, 09.X.2017, A. Cobos-Villagrán 645 (ENCB), 646 (ENCB); loc. cit., T. Raymundo 7005 (ENCB); carretera 75-D, San Luis - Cd. Valles km 98, 02.XI.2017, A. Cobos-Villagrán 772 (ENCB), 774 (ENCB). Municipio Rayón, Cerro al NE del cafetal, 29. XI.2012, I. Álvarez 319 (ENCB); carretera Cd. Valles - San Luis Potosí km 67, 02.XI.2017, A. Cobos-Villagrán 920 (ENCB). Municipio Tamasopo, carretera Río Verde - Cd. VaIles km 64, 01.XI.2007, T. Raymundo y R. Valenzuela 1905 (ENCB); El Sauz, km 28, 30.VII.2009, T. Raymundo 3266 (ENCB); loc. cit., 31.VIII.2009, J. Jaimes y H. Luna 26 (ENCB), 30 (ENCB); loc. cit., 12.X.2009, T. Raymundo s.n. (ENCB); loc. cit., T. Raymundo y R. Valenzuela s.n. (ENCB); carretera Río Verde - Cd. Valles km 70, 09.IX.2015, T. Raymundo 5805 (ENCB), 5819 (ENCB); loc. cit., R. Valenzuela 16241 (ENCB); Potrero del Hotel Real Kimera, ejido Los Cafetales, 03. IX.2017, A. Cobos-Villagrán 788 (ENCB), 793 (ENCB), 798 (ENCB), 801 (ENCB), 802 (ENCB), 803 (ENCB); Puente de Dios, Huasteca Potosina, 04.XI.2017, S. Bautista-Hernández 1001 (ENCB); rancho El Desengaño, Torrencillas, carretera Cd. Valles-Río Verde km 37-38, 04.X1.2017, A. CobosVillagrán 824 (ENCB), 825 (ENCB). Sonora, municipio Álamos, El Cajón, APFF Sierra Álamos - Río Cuchujaqui, 14.X.2013, D. Castro-Bustos 110 (ENCB), 118 (ENCB), 119 (ENCB), 120 (ENCB), 131 (ENCB); loc. cit., T. Raymundo 4801 (ENCB), 4802 (ENCB), 4810 (ENCB), 4817 (ENCB), 4822 (ENCB); Promontorios, APFF Sierra Álamos - Río Cuchujaqui, 07.X.2014, T. Raymundo 5411 (ENCB), 5414 (ENCB), 5443 (ENCB); loc. cit., R. Valenzuela 15808 (ENCB), 15819 (ENCB), 15828 (ENCB), 15829 (ENCB), 15831 (ENCB), 15833 (ENCB), 15847 (ENCB); loc. cit., 27.X.2018, A. CobosVillagrán 2067 (ENCB), 2068 (ENCB), 2069 (ENCB), 2070 (ENCB), 2071 (ENCB), 2087 (ENCB); loc. cit., T. Raymundo 8045 (ENCB); loc. cit., R. Valenzuela 18731 (ENCB); rancho La Sierrita, APFF Sierra Álamos - Río Cuchujaqui, 15.X.2013, E. Escudero-Leyva 253 (ENCB); loc. cit., 07.X.2014, T. Raymundo 5440 (ENCB); loc. cit., 08.X.2014, T. Raymundo 5466 (ENCB), 5477 (ENCB), 5478 (ENCB), 5483 (ENCB); loc. cit., R. Valenzuela 15856 (ENCB); loc. cit., 8.X.2018, S. Bautista-Hernández 968 (ENCB); loc. cit., A. Cobos-Villagrán 2083 (ENCB), 2084 (ENCB), 2085 (ENCB), 2086 (ENCB); loc. cit., T. Raymundo 8082 (ENCB), loc. cit., R. Valenzuela
18762 (ENCB); sobre camino a Los Cantiles, Rancho La Sierrita, APFF Sierra Álamos - Río Cuchujaqui, 09.X.2014, T. Raymundo 5523 (ENCB), 5532 (ENCB), 5533 (ENCB); loc. cit., R. Valenzuela 15901 (ENCB), 15903 (ENCB). Municipio Benjamín Hill, Las Ánimas, Planicie Central del Desierto Sonorense, 17.VIII.2009, T. Raymundo 3173 (ENCB). Municipio Carbó, San Luis, Desierto Sonorense, 17.VIII.2009, T. Raymundo 3140 (ENCB), 3168 (ENCB). Municipio San Javier, Carretera Yécora - La Colorada km 135, 03.X.2016, $R$. Valenzuela 16962 (ENCB), 16963 (ENCB), 16970 (ENCB), 16996 (ENCB); loc. cit., 05.X.2016, R. Valenzuela 17127 (ENCB), 17137 (ENCB). Municipio San Miguel Horcasitas, 19.X.2013, E. Escudero-Leyva 279 (ENCB), loc. cit., T. Raymundo 4864 (ENCB), 4872 (ENCB); loc cit., R. Valenzuela 15284 (ENCB). Tabasco, municipio Emiliano Zapata, UMA Los Manatíes, 19.VII.2014, T. Raymundo 5214 (ENCB); loc. cit., 20.VII.2014, E. Escudero-Leyva 613 (ENCB); loc. cit., T. Raymundo 5220 (ENCB); loc. cit., R. Valenzuela 15620 (ENCB), 15631 (ENCB). Tamaulipas, municipio Gómez Farías, La Florida, Reserva de la Biósfera El Cielo, 24.VI.2018, A. Cobos-Villagrán 1862 (ENCB); loc. cit., 25.VI.2018, A. Cobos-Villagrán 1891 (ENCB), 1892 (ENCB), 1893 (ENCB), 1900 (ENCB), 1902 (ENCB), 1903 (ENCB), 1909 (ENCB), 1910 (ENCB), 1912 (ENCB), 1913 (ENCB), 1914 (ENCB), 1915 (ENCB), 1916 (ENCB); loc. cit., 26.VI.2018, A. CobosVillagrán 1938 (ENCB); ejido El Azteca, pasando el río, Reserva de la Biósfera El Cielo, 27.VII.2018, A. Cobos-Villagrán 1978 (ENCB); loc. cit., U. Rodríguez s.n. (ENCB); Sendero a Alta Cima, 27.VI.2018, G. Dávila 168 (ENCB).

Hábitat: esta especie crece gregaria sobre madera en descomposición de diversas angiospermas (Acacia spp., Acacia cochliacantha Willd., Bougainvillea glabra Choisy, Celtis pallida Torr. y Helietta parvifolia (A. Gray) Benth.), en bosque tropical caducifolio y matorral xerófilo, desde 0 a 2475 m s.n.m., ocasionando una pudrición blanda.

Distribución: Taiwán (Chen y Hsieh, 1996); Cataluña, España (Sierra-López, 2006); Costa Rica, Estados Unidos de América, Puerto Rico (Murillo et al., 2009); Argentina, Brasil, China, Costa Rica, Cuba, Dominica, Estados Unidos de América, Filipinas, Francia, Ghana, India, Islas Cook, Jamaica, Japón, Kenia, Malasia, Micronesia, Nueva Guinea, 
Nueva Zelanda, Puerto Rico, Sierra Leona, Tanzania y Tonga (Almeida et al., 2014); Tailandia (Thambugala et al., 2016) e Islas Andamán, India (Niranjan et al., 2018). En México, Rhytidhysteron rufulum se ha citado anteriormente del estado de Sonora (Méndez-Mayboca et al., 2010), Oaxaca (Raymundo et al., 2014; Álvarez et al., 2016), de Chiapas, Guerrero, Nuevo León, Puebla, Querétaro, Quintana Roo, Sonora, Tabasco, Tamaulipas, Veracruz y Yucatán (Chacón et al., 2014), y Xalapa, Veracruz (López y García, 2017). Se cita por primera vez para los estados de Campeche, Colima, Estado de México, Hidalgo, Jalisco, Nayarit y San Luis Potosí.

Notas taxonómicas: Rhytidhysteron rufulum se caracteriza por presentar epitecio de color anaranjado-rojizo, que en presencia de $\mathrm{KOH}$ al $10 \%$ cambia a magenta y la reacción perdura. Su peridio es fuertemente estriado con células de textura isodiamétrica. Es la especie de mayor distribución, prácticamente en toda la región pantropical.

Rhytidhysteron thailandicum Thambug. \& K.D. Hyde, Cryptogamie Mycologie 37(1): 110. 2016. TIPO: TAILANDIA. Provincia de Chiang Rai, Centro de Investigación Hortícola Chiangrai, 2012, K. M. Thambugala 8 (holotipo: MFLU, isotipo: PDD). Fig. 4.

Histerotecios 1.0-1.5(-1.9) mm de largo, 0.4-0.6(-0.9) $\mathrm{mm}$ de ancho, $0.6-1.0 \mathrm{~mm}$ de alto $(\overline{\mathrm{X}}=1.53 \times 0.71 \times 0.78$ $\mathrm{mm}, \mathrm{n}=10$ ), en forma de conchas, globosos a subglobosos, lenticulares y deprimidos, negros, carbonosos, errumpentes, solitarios o dispersos, raramente gregarios, borde estriado, con surco o hendidura longitudinal irregular, sin embargo, no toma la forma apotecial en la madurez o con humedad; peridio de un grosor regular de 66.5-76 $\mu \mathrm{m}$, ensanchándose ligeramente hacia la parte basal hasta $95 \mu \mathrm{m}$, compuesto por capas de células pseudoparenquimáticas color marrón a marrón oscuro, de textura isodiamétrica o globulosa-angular con células de 10-12 × 5-7 $\mu \mathrm{m}$, paredes delgadas, $1 \mu \mathrm{m}$ de grosor; epitecio no visible, en reacción con $\mathrm{KOH}$ al $10 \%$ cambia a púrpura; pseudoparáfisis $3 \mu \mathrm{m}$ de ancho, capitadas, filamentosas, densas, hialinas, septadas, sin ramificar, fuertemente anastomosadas por encima de las ascas formando el epitecio; ascas bitunicadas, 170-185 $\times 12-13 \mu \mathrm{m}$, clavadas a cilíndricas, hialinas, uniseriadas o parcialmente superpuestas, octospóricas, con la base corta y recta; ascosporas 26-28(-30) $\times(9.6-) 10.4-11(-12) \mu \mathrm{m}$, $(\bar{x}=28 \times 10.6 \mu \mathrm{m}, \mathrm{n}=30)$, elipsoides, umbonadas en ambos extremos, amarillentas a pardas con tres septos transversales, cinguladas, pared gruesa y lisa.

Material estudiado: MÉXICO, Oaxaca, municipio San Mateo Peñasco, San Isidro, Dto. Tlaxiaco, 19.VI.2019, S. Bautista-Hernández s.n. (ENCB). Puebla, municipio San José Miahuatlán, Carretera Tehuacán - Cuicatlán km 76, Reserva de la Biósfera Tehuacán-Cuicatlán, 1.VI.2013, T. Raymundo 4564 (ENCB), 4565 (ENCB); loc. cit., 01.III.2014, $R$. Valenzuela 14963 (ENCB); loc. cit., 27.V.2016, T. Raymundo 6100 (ENCB), 6101, (ENCB); loc. cit., P. Reyes 30 (ENCB); loc. cit., R. Valenzuela 16601 (ENCB), 16605 (ENCB); loc. cit., 24.XI.2016, T. Raymundo 6343 (ENCB), 6344 (ENCB), 6345 (ENCB), 6346 (ENCB), 6347 (ENCB), 6348 (ENCB); loc. cit., R. Valenzuela 17070 (ENCB), 17071 (ENCB), 17073 (ENCB), 17074 (ENCB); loc. cit., 21.V.2017, J. Cuellar y D. Serrano 3 (ENCB); loc. cit., 22.V.2017, T. Raymundo 6646 (ENCB); loc. cit., R. Valenzuela 17270 (ENCB), 17271 (ENCB), 17273 (ENCB); loc. cit., 28.IV.2018, A. Cobos-Villagrán 970 (ENCB), 971 (ENCB), 972 (ENCB), 973 (ENCB), 974 (ENCB), 975 (ENCB). San Luis Potosí, municipio Guadalcázar, Carretera Cerritos - entronque a Tula, km 26, 09.X.2017, A. CobosVillagrán 645 (ENCB), 646 (ENCB); loc. cit., T. Raymundo 7005 (ENCB).

Hábitat: dispersos, sobre madera en descomposición de Acacia sp. y Helietta parvifolia en el matorral xerófilo, en regiones con clima árido y semiárido, en un gradiente altitudinal de 342 a 1882 m s.n.m., ocasionando una pudrición blanda.

Distribución: Chiang Rai Province, Tailandia (Thambugala et al., 2016) e Islas Andamán, India (Niranjan et al., 2018). En el presente estudio se registra por primera vez para México en las regiones fisiográficas Altiplanicie, Depresión del Balsas y Valle de Tehuacán-Cuicatlán.

Notas taxonómicas: esta especie se caracteriza por presentar los ascomas más pequeños del género, hasta 1.5 $\mathrm{mm}$ de largo. El material revisado concuerda con las des- 


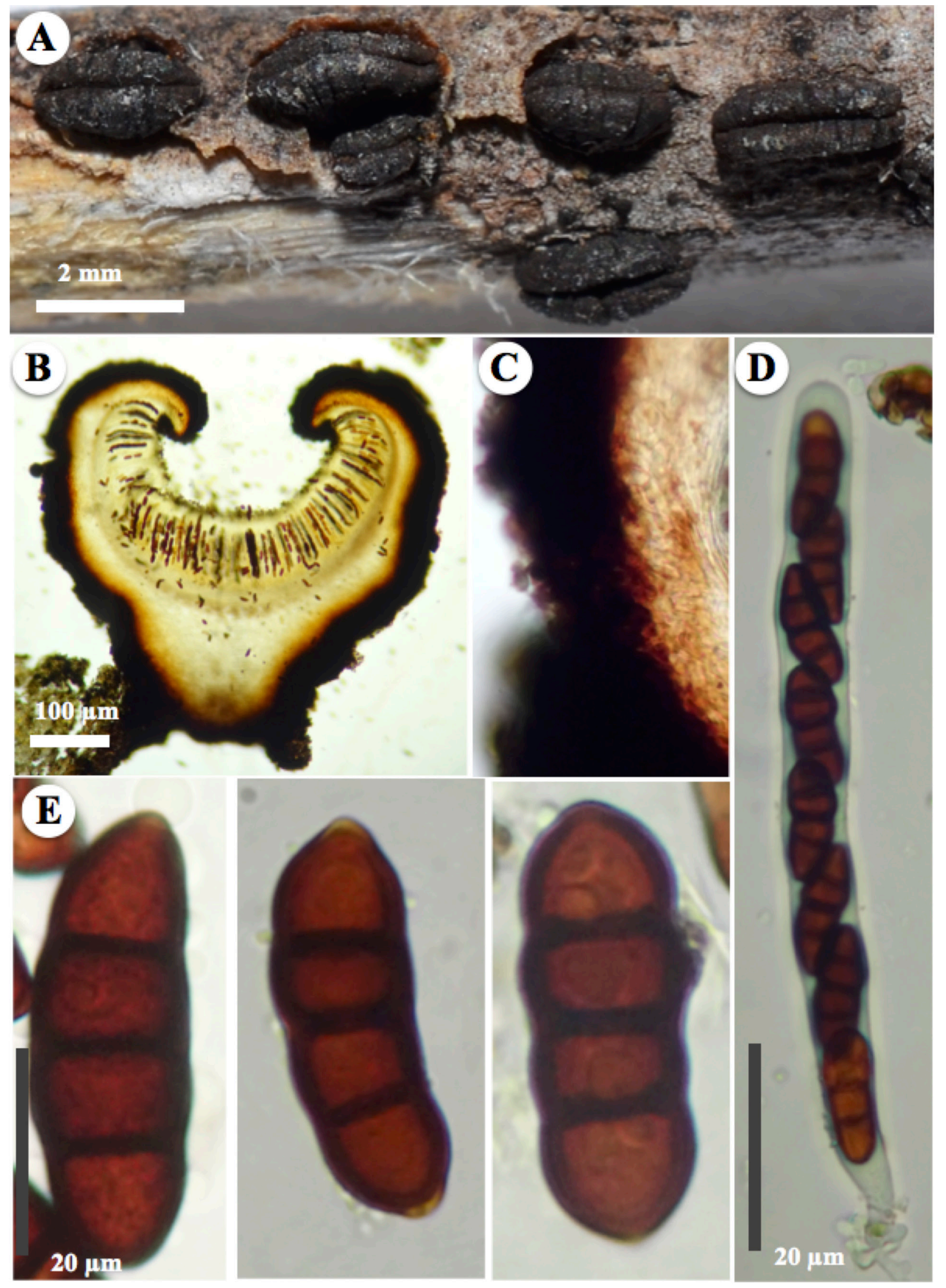

Figura 4: Rhytidhysteron thailandicum Thambug. \& K.D. Hyde. A. histerotecios; B. corte longitudinal del histerotecio; corte longitudinal del histerotecio; C. células del peridio; D. asca; E. ascosporas. 
cripciones previas de $R$. thailandicum, descrita de Tailandia y citada de la India (Thambugala et al., 2016; Niranjan et al., 2018).

\section{Discusión}

Las especies del género Rhytidhysteron han sido difíciles de determinar por las características morfológicas muy similares en valores de medidas en histerotecios, ascas y ascosporas que se solapan. Sin embargo, en este estudio se obtuvieron las medidas promedio de esas estructuras y los caracteres de importancia taxonómica de las especies de Rhytidhysteron en México (Cuadro 1). Uno de los primeros caracteres son el tamaño de los ascomas que varía de 1 a $5 \mathrm{~mm}$ de longitud, de tal forma, que $R$. neorufulum y $R$. rufulum presentan los histerotecios más grandes de hasta $2.5 \mathrm{~mm}$ de largo. Sin embargo, estos dos se pueden separar porque el primero presenta peridio liso y el segundo peridio fuertemente estriado. En contraste, $R$. thailandicum forma los histerotecios más pequeños con un promedio de $1.5 \mathrm{~mm}$ de largo.

Rhytidhysteron neohysterinum, $R$. neorufulum y $R$. rufulum presentan epitecio en tonos anaranjados a rojos que en presencia de $\mathrm{KOH}$ al $10 \%$ viran a violeta, morado o magenta, respectivamente. Dicha reacción suele ser muy rápida en las primeras dos especies volviéndose hialino, mientras que en $R$. rufulum la reacción perdura y en $R$. thailandicum no hay reacción al $\mathrm{KOH}$. En cuanto a la textura del peridio se diferencian dos tipos de acuerdo con Yacharoen et al. (2015): isodiamétrica o globulosa-angular e isoradiada o prismáticas. Del primer tipo están presentes en $R$. rufulum y $R$. thailandicum, del segundo en $R$. neorufulum y $R$. neohysterinum.

Respecto al tamaño de las ascosporas podemos apreciar las diferencias en las medias (que se obtuvieron de un total de 30 medidas de ascosporas), ya que los intervalos de tamaño siguen siendo un problema debido al solapamiento de estas medidas. Las ascosporas más grandes son de $R$. neorufulum, con un promedio de $32 \mu \mathrm{m}$ de largo, seguidas de $R$. thailandicum con $28 \mu \mathrm{m}$. A pesar de que los tamaños son muy parecidos, también hay diferencias en cuanto al ancho y la forma que tienen estas.

La distribución del género Rhytidhysteron se concentra en ecosistemas tropicales y secos en la región neotropi- cal de México, de tal manera que $R$. rufulum y $R$. neorufulum, con 247 especímenes estudiados (64.83\% del total), se localizaron en bosque tropical caducifolio entre 0 y 1900 $\mathrm{m}$ de altitud (Rzedowski, 2006). Esto coincide con Samuels y Müller (1979) y Thambugala et al. (2016), quienes indican que $R$. rufulum se distribuye en zonas tropicales. Cabe señalar que $R$. rufulum es una especie generalista, capaz de utilizar diferentes sustratos (Murillo et al., 2009), lo cual podría explicar su amplia frecuencia y distribución.

Rhytidhysteron thailandicum se encontró sobre ramas de Fabáceas, Acacia spp. y Helietta parvifolia, se distribuye en el matorral xerófilo del Valle de Tehuacán-Cuicatlán y del Altiplano Mexicano. Considerando que este ecosistema ocupa aproximadamente $40 \%$ de la superficie del país y por consiguiente es el más vasto de los tipos de vegetación (Rzedowski, 2006), podrían registrarse más especímenes en otras partes del país.

Los 208 (54.6\% del total) especímenes recolectados en este estudio, y los 173 (45.4\%) ejemplares de herbario resultan en un total de 381 , los cuales se localizan en 10 de las 17 provincias florísticas de acuerdo con Rzedowski (2006). De tal manera que la Costa Pacífica es la mejor representada con 127 especímenes (33.3\%), seguida de la Sierra Madre Oriental con 64 (16.8\%), el Altiplanicie con 58 (15.2\%), la Península de Yucatán y el Valle de TehuacánCuicatlán 31 (8.1\%) cada uno, la Costa del Golfo de México con 30 (7.9\%), Serranías Meridionales con 18 (4.7\%), Planicie Costera del Noroeste con 14 (3.7\%), Planicie Costera del Pacífico con 6 (1.6\%) y Depresión del Balsas 2 (0.5\%).

En el bosque tropical caducifolio se encontraron 249 especímenes (65.35\%), correspondientes a las especies $R$. rufulum y $R$. neorufulum; en el matorral xerófilo 103 especímenes (27.03\%), pertenecientes a las especies $R$. thailandicum, $R$. rufulum y $R$. neohysterinum. En mucha menor proporción en el bosque tropical perennifolio con 15 especímenes (4\%) de $R$. rufulum y $R$. neorufulum; en el bosque mesófilo de montaña cuatro especímenes (1.05\%) de $R$. rufulum; en los bosques templados (bosque de Pinus, PinusQuercus y Quercus) únicamente encontramos a $R$. rufulum con ocho ejemplares $(2.1 \%)$, y en vegetación perturbada con un ejemplar de $R$. rufulum y $R$. thailandicum el $0.52 \%$.

En la figura 5 se muestra la distribución de las especies de Rhytidhysteron en México, donde observamos que 


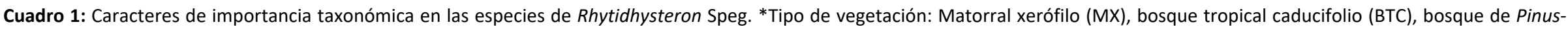
Quercus (BPQ).

\begin{tabular}{|c|c|c|c|c|c|c|c|c|c|}
\hline \multirow{2}{*}{ Especie } & \multicolumn{3}{|c|}{$\begin{array}{l}\text { Tamaño del } \\
\text { histerotecio } \\
\text { (mm) }\end{array}$} & \multicolumn{2}{|c|}{ Color del epitecio } & \multirow{2}{*}{$\begin{array}{c}\text { Células del } \\
\text { peridio }\end{array}$} & \multirow{2}{*}{$\begin{array}{c}\text { Tamaño } \\
\text { promedio } \\
\text { ascosporas } \\
(\mu \mathrm{m}) \\
\text { largo } \times \text { ancho } \\
(\mathrm{n}=30)\end{array}$} & \multirow{2}{*}{$\begin{array}{c}\text { Tipo de } \\
\text { vegetación }\end{array}$} & \multirow{2}{*}{$\begin{array}{c}\text { Región } \\
\text { fisiográfica }\end{array}$} \\
\hline & Forma & $\begin{array}{l}\text { largo, ancho, } \\
\text { alto } \\
(n=10)\end{array}$ & $\begin{array}{c}\text { Ornamentación } \\
\text { peridio }\end{array}$ & En fresco & KOH al 5\% & & & & \\
\hline $\begin{array}{l}\text { R. neohysterinum } \\
\text { Cobos-Villagrán, Hdz.- } \\
\text { Rdz., R. Valenz. \& } \\
\text { Raymundo }\end{array}$ & apotecioide & $\begin{array}{c}\bar{x}=1.97 \times 1.32 \times \\
0.9 \mathrm{~mm}\end{array}$ & estriado & naranja & morado & isoradiadas & $\begin{array}{c}\bar{x}=26.6 \times 9.4 \\
\mu \mathrm{m}\end{array}$ & MX, BTC & $\begin{array}{l}\text { Altiplanicie, } \\
\text { Sierra Madre } \\
\text { Oriental }\end{array}$ \\
\hline $\begin{array}{l}\text { R. neorufulum } \\
\text { Thambug. \& K.D. } \\
\text { Hyde }\end{array}$ & $\begin{array}{l}\text { navicular- } \\
\text { fusifrome }\end{array}$ & $\begin{array}{c}\bar{x}=2.48 \times 0.87 \times \\
1.01 \mathrm{~mm}\end{array}$ & liso & naranja & violeta & isodiamétricas & $\bar{x}=32 \times 11 \mu \mathrm{m}$ & BTC & $\begin{array}{c}\text { Costa del Golfo } \\
\text { de México, } \\
\text { Costa Pacífica, } \\
\text { Península } \\
\text { de Yucatán, } \\
\text { Sierra Madre } \\
\text { Oriental }\end{array}$ \\
\hline $\begin{array}{l}\text { R. rufulum (Spreng.) } \\
\text { Speg. }\end{array}$ & apotecioide & $\begin{array}{c}\bar{X}=2.0 \times 1.12 \times \\
0.94 \mathrm{~mm}\end{array}$ & estriado & $\begin{array}{c}\text { naranja-rojizo, } \\
\text { amarillo o } \\
\text { negro }\end{array}$ & magenta & isodiamétricas & $\overline{\mathrm{X}}=26 \times 8.8 \mu \mathrm{m}$ & BTC, MX & $\begin{array}{c}\text { Altiplanicie, } \\
\text { Costa del } \\
\text { Golfo de } \\
\text { México, Costa } \\
\text { Pacífica, } \\
\text { Depresión } \\
\text { del Balsas, } \\
\text { Península } \\
\text { de Yucatán, } \\
\text { Planicie } \\
\text { Costera del } \\
\text { Noroeste, } \\
\text { Serranías } \\
\text { Meridionales } \\
\text { Sierra Madre } \\
\text { Oriental }\end{array}$ \\
\hline $\begin{array}{c}\text { R. thailandicum } \\
\text { Thambug. \& K.D. } \\
\text { Hyde }\end{array}$ & lenticular & $\begin{array}{c}\bar{x}=1.53 \times 0.71 \times \\
0.78 \mathrm{~mm}\end{array}$ & estriado & No expuesto & púrpura & isodiamétricas & $\overline{\mathrm{x}}=8 \times 10.6 \mu \mathrm{m}$ & $M X$ & $\begin{array}{l}\text { Altiplanicie, } \\
\text { Depresión del } \\
\text { Balsas, Valle } \\
\text { de Tehuacán- } \\
\text { Cuicatlán }\end{array}$ \\
\hline
\end{tabular}




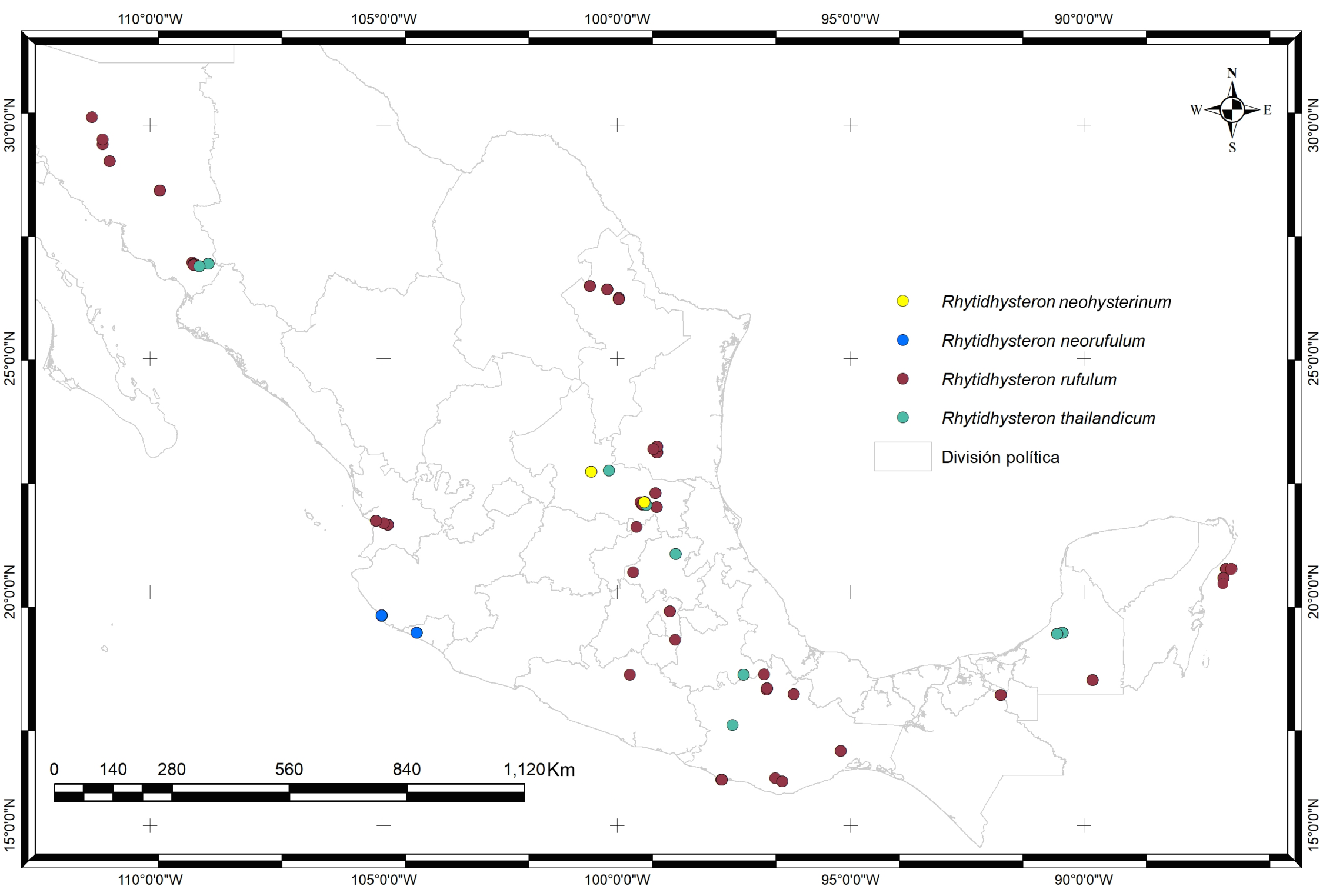

Figura 5: Mapa de distribución de las especies de Rhytidhysteron Speg. en México. 
$R$. rufulum es la especie más frecuente con 242 especímenes, que corresponde a $63.52 \%$ y con la mayor distribución en nueve de las 17 provincias, siguiendo el criterio de Rzedowski (2006): Costa Pacífica (78 ejemplares), Altiplanicie (51), Sierra Madre Oriental (46), Costa del Golfo de México (20), Península de Yucatán (15), Serranías Meridionales (15), Planicie Costera del Noreste (14), Planicie Costera del Pacífico (2) y Depresión del Balsas (1).

La mayoría de los especímenes se encontraron sobre ramas de árboles recién muertos o en descomposición, principalmente en géneros de la familia Fabaceae, donde observamos representantes de las especies $R$. neohysterinum, $R$. rufulum y $R$. thailandicum. Sobre representantes de la familia Rutaceae se observó a $R$. rufulum y $R$. thailandicum, y $R$. neorufulum se encuentra sobre Burseraceae. Rhytidhysteron rufulum es la especie que presentó más variabilidad de hospederos, en familias como Boraginaceae, Cannabaceae, Fabaceae, Nyctaginaceae y Rutaceae (Cuadro 2).

Generalmente las especies de Rhytidhysteron se describen como hongos saprobios o patógenos débiles que crecen en plantas leñosas en hábitats terrestres (Yacharoen et al., 2015). Sin embargo, recientemente Pudhom y Teerawatananond (2014), Chokpaiboon et al. (2016) y Siridechakorn et al. (2017) reportaron que Rhytidhysteron es un hongo endófito que produce metabolitos secundarios, conocidos como espirobisnaftalenos con diversas actividades biológicas. Por otra parte, el género también ha sido identificado como un patógeno oportunista que causa micosis subcutáneas, cromoblastomicosis y feohifomicosis en pacientes inmunosuprimidos (Chowdhary et al., 2008; Mishra et al., 2014; Mahajan et al., 2014; Chander et al., 2016).

\section{Conclusiones}

En Rhytidhysteron, los caracteres macromorfológicos con mayor peso taxonómico para diferenciar a las especies son el tamaño de los histerotecios y la reacción del epitecio en $\mathrm{KOH}$ al $10 \%$, mientras que los microscópicos son la textura del peridio y el tamaño de las ascosporas.

La mayoría de las especies de Rhytidhysteron en México tienen una distribución en ecosistemas tropicales y secos, en un gradiente altitudinal de los 0 a los $1600 \mathrm{~m}$, aunque la mayoría se concentra a nivel de mar. Las especies de Rhytidhysteron se encuentran principalmente en el bosque tropical caducifolio con más de $60 \%$ de los especímenes, lo que podría indicar que en este tipo de vegetación es donde mejor se ha adaptado el grupo. Rhytidhysteron rufulum es una especie abundante con amplia distribución en bosque tropical caducifolio y matorral xerófilo de México; se ubica en toda la región neotropical, principalmente en la Planicie Costera del Pacífico. La mayoría de los especímenes se encontraron sobre ramas de árboles recién muertos o en descomposición, principalmente en géneros de la familia Fabaceae.

\section{Contribución de autores}

$A C V, R V, R P C M$ y TR concibieron y diseñaron el estudio; $A C V, R V, M M P, T R$ realizaron las recolecciones y determinaciones de las especies; ACV, CHR, LVT, LEMC, RPCV y TR contribuyeron a la adquisición de datos para el trabajo. Las fotos de las figuras fueron tomadas por ACV, RVG y TR. ACV escribió el manuscrito con la ayuda de TR. Todos los autores contribuyeron a la discusión, revisión y aprobación del manuscrito final.

\section{Financiamiento}

Este estudio fue apoyado por el Instituto Politécnico Nacional (IPN) a través de la Secretaría de Investigación y Posgrado (SIP) en los proyectos: 20195221, 20195222, 20200248, 20200956. Se agradece el apoyo financiero para las recolecciones que se hicieron en los estados de Tamaulipas, Jalisco y Quintana Roo por parte del proyecto 252934 SEP-CONACyT de la Convocatoria de Ciencia Básica. ACV fue becaria de Consejo Nacional de Ciencia y Tecnología (CONACyT) para realizar sus estudios de Maestría en Biociencias en la Escuela Nacional de Ciencias Biológicas (ENCB) del IPN y contó con la beca de Estímulo Institucional de Formación de Investigadores (BEIFI). RPCV agradece a CONACYT por la beca posdoctoral. CHR, LVT, RV, TR, LEMC recibieron becas para realizar sus investigaciones de la Comisión de Operación y Fomento de las Actividades Académicas del IPN, la Beca EDI de la Secretaria de Posgrado e Investigación del IPN, y del Sistema Nacional de Investigadores.

\section{Agradecimientos}

Los autores agradecen a las autoridades de la Escuela Nacional de Ciencias Biológicas (ENCB) del Instituto Politécni- 


\begin{tabular}{|c|c|c|c|c|c|}
\hline Especie & Espécimen & Hospedero & Familia & Región fisiográfica & $\begin{array}{c}\text { Tipo de } \\
\text { vegetación }^{*}\end{array}$ \\
\hline $\begin{array}{c}\text { Rhytidhysteron neohysterinum } \\
\text { Cobos-Villagrán, R. Valenz. \& } \\
\text { Raymundo }\end{array}$ & $\begin{array}{l}\text { A. Cobos-Villagrán } 773 \text { (ENCB), } 776 \text { (ENCB), } 777 \\
\text { (ENCB) }\end{array}$ & Acacia sp. Mill. & Fabaceae & Altiplanicie & $M X$ \\
\hline $\begin{array}{l}\text { Rhytidhysteron neorufulum } \\
\text { Thambug. \& K.D. Hyde }\end{array}$ & $\begin{array}{l}\text { A. Camargo s.n (ENCB); R. Valenzuela } 16717 \\
\text { (ENCB), } 16718 \text { (ENCB), } 16719 \text { (ENCB), } 16729 \\
\text { (ENCB), } 16732 \text { (ENCB), } 16747 \text { (ENCB) }\end{array}$ & $\begin{array}{l}\text { Bursera sp. } \\
\text { Jacq. ex L. }\end{array}$ & Burseraceae & Costa Pacífica & BTC \\
\hline $\begin{array}{l}\text { Rhytidhysteron rufulum (Spreng.) } \\
\text { Speg. }\end{array}$ & $\begin{array}{l}\text { A. Cobos-Villagrán } 688 \text { (ENCB), } 689 \text { (ENCB), } 690 \\
\text { (ENCB), } 691 \text { (ENCB), } 692 \text { (ENCB), } 695 \text { (ENCB), } \\
696 \text { (ENCB), } 697 \text { (ENCB) }\end{array}$ & $\begin{array}{l}\text { Acacia sp. } \\
\text { Mill. }\end{array}$ & Fabaceae & Altiplanicie & $\mathrm{MX}$ \\
\hline $\begin{array}{l}\text { Rhytidhysteron rufulum (Spreng.) } \\
\text { Speg. }\end{array}$ & $\begin{array}{l}\text { A. Cobos-Villagrán } 2069 \text { (ENCB), } 2070 \text { (ENCB), } \\
2087(\text { ENCB) }\end{array}$ & $\begin{array}{c}\text { Acacia cochleacantha } \\
\text { Willd. }\end{array}$ & Fabaceae & Costa Pacífica & $\mathrm{MX}$ \\
\hline $\begin{array}{l}\text { Rhytidhysteron rufulum (Spreng.) } \\
\text { Speg. }\end{array}$ & $\begin{array}{l}\text { A. Cobos-Villagrán } 682 \text { (ENCB), } 685 \text { (ENCB), } 686 \\
\text { (ENCB) }\end{array}$ & $\begin{array}{l}\text { Bougainvillea glabra } \\
\text { Choisy }\end{array}$ & Nyctaginaceae & Antiplanicie & $M X$ \\
\hline $\begin{array}{l}\text { Rhytidhysteron rufulum (Spreng.) } \\
\text { Speg. }\end{array}$ & $\begin{array}{c}\text { Cobos-Villagrán } 715 \text { (ENCB), } 718 \text { (ENCB), } 732 \\
\text { (ENCB), } 751 \text { (ENCB), } 752 \text { (ENCB); T. Raymundo } \\
7029 \text { (ENCB) }\end{array}$ & Celtis pallida Torr. & Cannabaceae & Antiplanicie & $\mathrm{MX}$ \\
\hline $\begin{array}{l}\text { Rhytidhysteron rufulum } \\
\text { (Spreng.) Speg. }\end{array}$ & $\begin{array}{c}\text { A. Cobos-Villagrán } 824 \text { (ENCB), } 825 \text { (ENCB); S. } \\
\text { Bautista-Hernández s.n. (ENCB). }\end{array}$ & $\begin{array}{c}\text { Ehretia anacua (Terán \& } \\
\text { Berland.) I.M. Johnst. }\end{array}$ & Boraginaceae & $\begin{array}{c}\text { Mesoaméricana de } \\
\text { Montaña }\end{array}$ & BTC \\
\hline $\begin{array}{l}\text { Rhytidhysteron rufulum (Spreng.) } \\
\text { Speg. }\end{array}$ & $\begin{array}{l}\text { A. Cobos-Villagrán } 741 \text { (ENCB), } 750 \text { (ENCB), } 757 \\
\text { (ENCB), } 762 \text { (ENCB) }\end{array}$ & $\begin{array}{l}\text { Helietta parvifolia (A. } \\
\text { Gray) Benth. }\end{array}$ & Rutaceae & Antiplanicie & MX, BTC \\
\hline $\begin{array}{l}\text { Rhytidhysteron thailandicum } \\
\text { Thambug. \& K.D. Hyde }\end{array}$ & $\begin{array}{c}\text { A. Cobos-Villagrán } 645 \text { (ENCB), } 646 \text { (ENCB), } 970 \\
\text { (ENCB), } 971 \text { (ENCB), } 972 \text { (ENCB), } 973 \text { (ENCB), } \\
974 \text { (ENCB), } 975 \text { (ENCB); J. Cuellar y D. Serrano } \\
3 \text { (ENCB); T. Raymundo } 7005 \text { (ENCB); P. Reyes } \\
30 \text { (ENCB) }\end{array}$ & $\begin{array}{l}\text { Helietta parvifolia (A. } \\
\text { Gray) Benth. }\end{array}$ & Rutaceae & Antiplanicie & $\mathrm{MX}$ \\
\hline $\begin{array}{l}\text { Rhytidhysteron thailandicum } \\
\text { Thambug. \& K.D. Hyde }\end{array}$ & $\begin{array}{l}\text { T. Raymundo } 4564 \text { (ENCB), } 4565 \text { (ENCB), } 6100 \\
\text { (ENCB), } 6101 \text { (ENCB), } 6343 \text { (ENCB), } 6344 \\
\text { (ENCB), } 6345 \text { (ENCB), } 6346 \text { (ENCB), } 6347 \\
\text { (ENCB), -6348 (ENCB), } 6646 \text { (ENCB); } R . \\
\text { Valenzuela } 16601 \text { (ENCB), } 16605 \text { (ENCB), } \\
17070 \text { (ENCB), } 17074 \text { (ENCB), } 17270 \text { (ENCB), } \\
17271 \text { (ENCB), } 17272 \text { (ENCB), } 17273 \text { (ENCB) }\end{array}$ & Acacia sp. Mill. & Fabaceae & $\begin{array}{l}\text { Valle de Tehuacán- } \\
\text { Cuicatlán }\end{array}$ & BTC \\
\hline
\end{tabular}


co Nacional las facilidades otorgadas para realizar el presente estudio.

\section{Literatura citada}

Almeida, D. A. C., L. F. P. Gusmão y A. N. Miller. 2014. Brazilian Semi-Arid Ascomycetes I: New and interesting records of hysteriaceous ascomycetes. Mycosphere 5(2): 379-391. DOI: http://dx.doi.org/10.5943/mycosphere/5/2/11

Álvarez, V. I., T. Raymundo y R. Valenzuela. 2016. Hongos histeroides del Bosque Tropical Caducifolio en el Parque Nacional Lagunas de Chacahua, Oaxaca, México. Acta Botanica Mexicana 116: 49-64. DOI: https://doi. org/10.21829/abm116.2016.1118

Boehm, E. W., C. L. Schoch y J. W. Spatafora. 2009a. On the evolution of the Hysteriaceae and Mytilinidiaceae (Pleosporomycetidae, Dothideomycetes, Ascomycota) using four nuclear genes. Mycological Research 113(4): 461-479. DOI: https://dx.doi.org/10.1016/j.mycres.2008.12.001.

Boehm, E. W., G. K. Mugambi, A. N. Miller, S. M. Huhndorf, S. Marincowitz, J. W. Spatafora y C. L. Schoch. 2009b. A molecular phylogenetic reappraisal of the Hysteriaceae, Mytilinidiaceae and Gloniaceae (Pleosporomycetidae, Dothideomycetes) with keys to the world species. Studies in Mycology 64: 49-83. DOI: https://dx.doi.org/10.3114/ sim.2009.64.03.

Chacón, S., F. Tapia y M. Esqueda. 2014. New records of Dothideomycetes from Mexico. Mycotaxon 128: 145-157. DOI: https://dx.doi.org/10.5248/128.145

Chander, J., N. Singla, R. Kundu, U. Handa y A. Chowdhary. 2016. Phaeohyphomycosis caused by Rhytidhysteron rufulum and review of literature. Mycopathologia 182: 403-407. DOI: http://dx.doi.org/10.1007/s11046-016-0064-x

Chen, C. Y. y W. H. Hsieh. 1996. Two new species and some new records of ascomycetes from Taiwan. Botanical Bulletin of Academia Sinica 37: 219-227.

Chokpaiboon, S., S. Choodej, N. Boonyuen, T. Teerawatananond y K. Pudhom. 2016. Highly oxygenated chromones from mangrove-derived endophytic fungus Rhytidhysteron rufulum. Phytochemistry 122: 172-177. DOI: http://dx.doi. org/10.1016/j.phytochem.2015.12.010

Chowdhary, A., J. Guarro, H. S. Randhawa, J. Gené, J. Cano, R. K. Jain, S. Kumar y G. Khanna. 2008. A rare case of chromoblastomycosis in a renal transplant recipient caused by a non-sporulating species of Rhytidhysteron. Medical Mycology 46: 163-166. DOI: http://dx.doi. org/10.1080/13693780701630420

Kirk, P. M., P. F. Cannon, D. W. Minter y J. A. Stalpers. 2008. Ainsworth \& Bisby's Dictionary of the Fungi. 10th ed. CAB International. Wallingford, UK. 771 pp.

Kumar, V., R. Cheewangkoon, K. M. Thambugala, G. E. B. Jones, R. Brahmanage, M. Doilom, R. Jeewon y K. Hyde. 2019. Rhytidhysteron mangrovei (Hysteriaceae), a new species from mangroves in Phetchaburi Provinces, Thailand. Phytotaxa 401(3): 166-178 DOI: https://doi.org/10.11646/ phytotaxa.401.3.2

López, A. y J. García. 2017. Rhytidhysteron rufulum, Patellariales: Patellariaceae. Funga Veracruzana 151: 1-4.

Mahajan, V. K., V. Sharma., N. Prabha., K. Thakur, N. L. Sharma, S. M. Rudramurthy, P. S. Chauhan, K. S. Mehta y C. Abhinav. 2014. A rare case of subcutaneous phaeohyphomycosis caused by a Rhytidhysteron species: a clinico-therapeutic experience. International Journal of Dermatology 53: 14851489. DOI: http://dx.doi.org/10.1111/ijd.12529

Méndez-Mayboca, F., S. Chacón, M. Esqueda y M. L. Coronado. 2008. Ascomycetes of Sonora, Mexico. 1: The Ajos-Bavispe National Forest Reserve and Wildlife Refuge. Mycotaxon 103: 87-95.

Méndez-Mayboca, F., J. Checa, M. Esqueda y S. Chacón. 2010. New records of Loculoascomycetes from natural protected areas in Sonora, Mexico. Mycotaxon 111: 19-30. DOI: http:// dx.doi.org/10.5248/111.19

Merino, A. D. y S. Tello-Mora. 2013. Aportaciones Micológicas 17 Micobotánica-Jaén VIII(4): 1-4.

Mishra, K., S. Das., S. Goyal, C. Gupta, G. Rai, M. A. Ansari, R. Saha y A. Singal. 2014. Subcutaneous mycoses caused by Rhytidhysteron species in an immunocompetent patient. Medical Mycology Case Reports 5: 32-34. DOI: http:// dx.doi.org/10.1016/j.mmcr.2014.07.002

Murillo, C., F. J. Albertazzi, J. Carranza, H. T. Lumbsch y G. Tamayo. 2009. Molecular data indicate that Rhytidhysteron rufulum (Ascomycetes, Patellariales) in Costa Rica consists of four distinct lineages corroborated by morphological and chemical characters. Mycological Research 113: 405-416. DOI: http://dx.doi.org/10.1016/j.mycres.2008.09.003

Niranjan, M., S. Tiwari, A, Baghela y V. V. Sarma. 2018. New records of Ascomycetous fungi from Andaman Islands, 
India and their molecular sequence data. Current Research in Environmental y Applied Mycology 8(3): 331-350. DOI: http://dx.doi.org/10.5943/cream/8/3/5

Pudhom, K y T. Teerawatananond. 2014. Rhytidenones A-F, spirobisnaphthalenes from Rhytidhysteron sp. AS21B, an endophytic fungus. Journal of Natural Products 77(8): 19621966. DOI: http://dx.doi.org/10.1021/np500068y.

Raymundo, T., E. Escudero-Leyva, I. Ortega-López, D. CastroBustos, H. León-Avendaño y R. Valenzuela. 2014. Ascomicetos del Bosque Tropical Caducifolio en el Parque Nacional Lagunas de Chacahua, Oaxaca, México. Boletín de la Sociedad Micológica de Madrid 38: 9-21.

Rzedowski, J. 2006. Vegetación de México. 1a. Ed digital, Comisión Nacional para el Conocimiento y Uso de la Biodiversidad. México, D.F., México. 504 pp.

Samuels, G. J. y E. Müller. 1979. Life-history studies of Brazilian Ascomycetes. Rhytidhysteron rufulum and the genus Eutryblidiella. Sydowia 32: 277-292.

Sierra-López, D. 2006. Contribución al estudio de los ascomicetes bitunicados de Cataluña. Acta Botánica Barcinonensia 50: 5-434.

Siridechakorn, I., Z. Yue, Y. Mittraphab, X. Lei y K. Pudhom. 2017. Identification of spirobisnaphthalene derivatives with anti- tumor activities from the endophytic fungus Rhytidhysteron rufulum AS21B. Bioorganic \& Medicinal Chemistry 25(11): 2878-2882. DOI: http://dx.doi.org/10.1016/j. bmc.2017.02.054.

Soto-Medina, E.y R. Lücking. 2017. A new species of Rhytidhysteron (Ascomycota: Patellariaceae) from Colombia, with a provisional working key to known species in the world. Revista de la Academia Colombiana de Ciencias Exactas, Físicas y Naturales 41(158): 59-63. DOI: https://dx.doi. org/10.18257/raccefyn.423

Spegazzini, C. 1881. Fungi argentini additis nonnullis brasiliensibus montevideensibusque. Pugillus quartus (Continuación). Anales de la Sociedad Científica Argentina 12: 174-189.

Thambugala, K. M., K. D. Hyde, P. D. Eungwanichayapant, A. I. Romero y Z. Y. Liu. 2016. Additions to the genus Rhytidhysteron in Hysteriaceae. Cryptogamie Mycologie 37: 99-116. DOI: https://dx.doi.org/10.7872/crym/v37. iss1.2016.99

Yacharoen S., Q. Tian, P. Chomnunti, S. Boonmee, E. Chukeatirote, J. D. Bhat y K. D. Hyde. 2015. Patellariaceae revisited. Mycosphere 6: 290-326. DOI: https://dx.doi.org/10.5943/ mycosphere/6/3/7 